\title{
Interpretation of the cause for the expansion of universe having its correlation with the cosmological constant $(\Lambda)$ through the consciousness model
}

\author{
Dhananjay Pal \\ Email address: \\ dhananjay.pal123@gmail.com,paldhananjay46@yahoo.com
}

Pharmacy College, Bengal School of Technology, Sugandha-Delhi Road, Chuchura, Dist.-Hooghly, West Bengal, INDIA

\section{To cite this article:}

Dhananjay Pal. Interpretation of the Cause for the Expansion of Universe Having Its Correlation with the Cosmological Constant ( $\Lambda$ ) through the Consciousness Model. American Journal of Physics and Applications. Vol. 1, No. 2, 2013, pp. 45-49.

doi: 10.11648/j.ajpa.20130102.13

\begin{abstract}
A single field emerged at the origin of the universe, already containing within itself the blueprint of the physical universe. The primordial single field triggered the onset of the universe. Most physicists believe that a single super-force dominated the first instants of creation. Scientists have arrived at a simple but decisive conclusion that consciousness is very much a part of the universe, like other objects. Our consciousness model involving thought-carrying particle (TCP), thought retaining particle (TRP) and thought force $\left(\mathrm{T}_{\mathrm{F}}\right)$ signifies the existence of universal consciousness that exists along with the universe. This universal consciousness is a functional state of the universal mind. This universal mind (UM) is evolved at the Big Bang from void. The UM is constituted by these TCP and TRP in the inherent presence of thought force $\left(\mathrm{T}_{\mathrm{F}}\right)$. Thought force $\left(\mathrm{T}_{\mathrm{F}}\right)$ is an expression of universal consciousness. The Thought force $\left(\mathrm{T}_{\mathrm{F}}\right)$ being the primordial quantum field functions as the original super-force. $\mathrm{T}_{\mathrm{F}}$ being the original super-force functions as the origin of all the fundamental fields. TCP is the carrier of thought force $\left(\mathrm{T}_{\mathrm{F}}\right)$ that, in turn, appears to be the origin of all the fields. The quantized energy $\left(\varepsilon_{T}\right)$ of TCP is responsible to cause the universal consciousness as well as the cosmic microwave background radiation temperature. The individual consciousness owes its origin to the universal consciousness created by the same $\varepsilon_{T}$. The same $\varepsilon_{T}$ is the energy responsible for generating thought force $\left(\mathrm{T}_{\mathrm{F}}\right)$. $\mathrm{T}_{\mathrm{F}}$ being an expression of the universal consciousness is applicable to any inanimate object as well as to any biological system (having thinking ability). The $\mathrm{T}_{\mathrm{F}}$ exerts its functions both in vitro and in vivo. We showed the existence of thought force in microcosm $\left[\mathrm{T}_{\mathrm{F}}(\right.$ micro) $)$ and thought force in macrocosm $\left[\mathrm{T}_{\mathrm{F}}(\mathrm{macro})\right]$. This $\mathrm{T}_{\mathrm{F}}$ (micro) is theoretically found to be stronger than the strong nuclear force. $\mathrm{T}_{\mathrm{F}}$ (macro) is theoretically found to be weaker even than the gravitational force. Recession velocity is found to be maintained by $\mathrm{T}_{\mathrm{F}}$ (macro). The upper limit of the cosmological constant agrees well with the value of $\mathcal{E}_{T}$ (= quantized energy of TCP).
\end{abstract}

Keywords: Universal Mind (UM), Cosmic Microwave Background Radiation (CMBR), Thought Force $\left(\mathrm{T}_{\mathrm{F}}\right), \mathrm{T}_{\mathrm{F}}(\mathrm{Micro})$, $\mathrm{T}_{\mathrm{F}}$ (Macro), Though-Carrying Particle (TCP), Thought Retaining Particle (TRP),

Quantized Energy $\left(\varepsilon_{T}\right)$ of TCP

\section{Introduction}

Consciousness model of Pal et al [1-3] involving TCP, TRP and thought force $\left(\mathrm{T}_{\mathrm{F}}\right)$ signifies the existence of universal consciousness that exists along with the universe. Pal et al [3] showed that the functional state of Universal Mind (UM) is the universal consciousness that exists along with the universe. Pal et al [3] explained that the UM is evolved at the Big Bang from the eternal Void. This Void, in turn, is the source of infinite energy. And this UM is a finer matter. The individual mind being a constituent of the UM is also a finer matter. The constituents of the UM and individual mind are the same. The ultimate constituents of matter and mind are the same as both mind and matter are aspects of one fundamental reality, which is called UM. The brain is the mediating link or interface between the individual mind and body.

Pal et al [3] explained that the constituents of the UM are the ultimate constituents of matter itself as everything in this universe is a manifestation of this UM. Pal et al [3] expressed that the UM is constituted by these TCP and TRP in the inherent presence of thought force $\left(\mathrm{T}_{\mathrm{F}}\right)$. Pal et al $[1,3]$ 
further explained that the ultimate constituents of matter and mind are these TCP and TRP in the inherent presence of thought force $\left(\mathrm{T}_{\mathrm{F}}\right)$ in vitro and thought force $\left(\mathrm{T}_{\mathrm{F}}\right)$ in vivo. Pal [4] explained the existence of the thought force $\left(\mathrm{T}_{\mathrm{F}}\right)$ that is an expression of the universal consciousness. Thought force $\left(\mathrm{T}_{\mathrm{F}}\right)$ is the primordial quantum field.

TCP cannot exist without TRP and vice versa. Many physicists believe that unifying all the forces, including gravity, into a single theory would require a phenomenon called super-symmetry. With super-symmetry, every fermion would have a boson twin, and vice-versa. TCP that behaves like boson should accompany its super-symmetrical partner TRP that functions like fermion in the generalized simpler way. Thus TCP like boson cannot have anti-particle. But TRP that functions like fermion should have its anti-particle and here it is shown as Anti-TRP. It is to be noted that these TCP and TRP function like wavicle: wave-particle duality.

\section{Thought Force}

Pal et al [1, 3] and Pal [4] explained that the quantized energy $\left(\varepsilon_{T}\right)$ of TCP is the energy responsible for generating thought force $\left(\mathrm{T}_{\mathrm{F}}\right)$; thus the $\mathrm{T}_{\mathrm{F}}$ may be expressed as

$$
\mathrm{T}_{\mathrm{F}}=\varepsilon_{T} / \mathrm{Di}
$$

where $\mathrm{D}_{\mathrm{i}}=$ Interacting distance.

The thought force $\left(\mathrm{T}_{\mathrm{F}}\right)$ has been postulated to be carried by TCP in the inherent presence of TRP. The TCP being the carrier of the $T_{F}$ would behave like bosons when TRP would function like fermions.

Depending on the $\mathrm{D}_{\mathrm{i}}$ (= Interacting distance), it is possible to calculate and identify two new forces viz. $\mathrm{T}_{\mathrm{F}}$ (micro) [= Thought force in microcosm $]$ and $\mathrm{T}_{\mathrm{F}}$ (macro) [= Thought force in macrocosm], the existence of which is indicated and expressed by $\mathrm{Pal}[4]$.

Pal [4] explained that Thought force $\left(\mathrm{T}_{\mathrm{F}}\right)$, an expression of the universal consciousness, is the primordial quantum field that, in turn, functions as the primary unified field. This $\mathrm{T}_{\mathrm{F}}$ being an expression of the universal consciousness is applicable to any inanimate object as well as to any biological system (having thinking ability). Thus the $\mathrm{T}_{\mathrm{F}}$ being an expression of the universal consciousness exerts its functions both in vitro and in vivo.

Physicists determined that underlying quantum fields give birth to elementary particles. Pal [4] expressed that the thought force $\left(\mathrm{T}_{\mathrm{F}}\right)$ is the primordial quantum field. Thought force $\left(T_{F}\right)$ being the primordial quantum field functions as the primary unified field. Thought force $\left(\mathrm{T}_{\mathrm{F}}\right)$ being the primordial quantum field gives birth to TRP that appears to be the origin of all the matter particles. TCP is the carrier of thought force $\left(\mathrm{T}_{\mathrm{F}}\right)$ that, in turn, appears to be the origin of all the fields. TCP thus appears to be the origin of all the field particles.

In a purpose to involve both the non-living and living systems of the world, Pal [4] has shown the existences of these TCP, TRP and thought force $\left(\mathrm{T}_{\mathrm{F}}\right)$ in vitro and thought force $\left(\mathrm{T}_{\mathrm{F}}\right)$ in vivo. Anyone can call this TCP by any other name, but as the highly developed living system will have to be evolved in the universe in the long run and as the thought of highly developed living system appears to be a kind of force to be called the thought force $\left(\mathrm{T}_{\mathrm{F}}\right)$ in vivo, we considered it is wise to call it as TCP. Further, as the universe exists along with the universal consciousness that, in turn, is created by the quantized energy $\left(\varepsilon_{T}\right)$ of TCP, we had to use the term TCP.

Pal (4) expressed that the non-living system of the world is governed by the thought force $\left(\mathrm{T}_{\mathrm{F}}\right)$ in vitro and this Thought force $\left(\mathrm{T}_{\mathrm{F}}\right)$ in vitro gives rise to $\mathrm{T}_{\mathrm{F}}$ (micro), $\mathrm{SNF}$, EMF, WNF, GF and $\mathrm{T}_{\mathrm{F}}$ (macro)

where $\mathrm{T}_{\mathrm{F}}$ (micro) $=$ Thought force in microcosm, $\mathrm{SNF}=$ Strong nuclear force, $\mathrm{EMF}=$ Electromagnetic force, $\mathrm{WNF}=$ Weak nuclear force, $\mathrm{GF}=$ Gravitational force and $\mathrm{T}_{\mathrm{F}}$ (macro) $=$ Thought force in macrocosm. It is to be noted here that $\mathrm{T}_{\mathrm{F}}$ (micro) is a stronger force than the SNF and $\mathrm{T}_{\mathrm{F}}$ (macro) is a weaker force even than the GF.

$\mathrm{Pal}$ (4) also expressed that the living system of the world is governed by the thought force $\left(\mathrm{T}_{\mathrm{F}}\right)$ in vivo and this Thought force $\left(\mathrm{T}_{\mathrm{F}}\right)$ in vivo is a type of force that represents the biological 'thought' which is the action of mind. This 'thought' being a type of force controls the 'thought processes' involving the firing of neurons through the quantum mechanical activities of these TCP and TRP in the presence of consciousness. This consciousness, in turn, is the quantized energy $\left(\varepsilon_{T}\right)$ of TCP. The thought force $\left(\mathrm{T}_{\mathrm{F}}\right)$ in vivo is demonstrated in numerous experiments in which thought has an effect on a physical process (often known as mind over matter). This biological 'thought' is a type of force that can cause movement. Controlling movement through thought alone is observed in several experiments conducted by many scientists as indicated by Pal (4). These experiments thus signify the existence of thought force $\left(\mathrm{T}_{\mathrm{F}}\right)$ in vivo.

Pal [4] explained the existence of $\mathrm{T}_{\mathrm{F}}$ (micro) (= Thought force in microcosm). This $\mathrm{T}_{\mathrm{F}}$ (micro) is the strongest interaction (a new class of 'extra strong' interaction) indicated by Weinberg [5] and Quigg [6]. It is stronger than SNF (Strong Nuclear Force). And this $\mathrm{T}_{\mathrm{F}}$ (micro) confirms the existence of TCP in the inherent presence of TRP in the microcosm.

Pal [4] has also shown the existence of $\mathrm{T}_{\mathrm{F}}$ (macro) $(=$ Thought force in macrocosm). It is the "weakest force" which is much weaker even than the gravity as explained by Perkins [7] and indicated by Weinberg [5]. This $\mathrm{T}_{\mathrm{F}}$ (macro) confirms the existence of TCP in the inherent presence of TRP into the quantum geometry of the universe.

\section{Cosmological Constant $(\Lambda)$}

The cosmological constant is a constant term in field equations of general relativity, represented by the Greek symbol Lambda, which allowed for a static universe. Later evidence supported the fact that the universe was indeed expanding and the cosmological constant was believed to be zero. Evidence in the late 1990s has begun supporting the idea that the universe is not only expanding, but that the 
expansion rate is actually accelerating. This meant that the cosmological constant wasn't just zero, as expected, but had to have a very slight positive value. The theory that has grown up around this positive cosmological constant is the theory of dark energy.

The simplest explanation for dark energy is that it is simply the "cost of having space": that is, a volume of space has some intrinsic, fundamental energy. This is the cosmological constant, sometimes called Lambda after the Greek letter $\Lambda$, the symbol used to mathematically represent this quantity. Since energy and mass are related by $E=m c^{2}$, Einstein's theory of general relativity predicts that it will have a gravitational effect. It is sometimes called a vacuum energy because it is the energy density of empty vacuum. In fact, most theories of particle physics predict vacuum fluctuations that would give the vacuum this sort of energy. This is related to the Casimir Effect, in which there is a small suction into regions where virtual particles are geometrically inhibited from forming (e.g. between plates with tiny separation). The cosmological constant is estimated by cosmologists to be on the order of $10^{-29} \mathrm{~g} / \mathrm{cm}^{3}$, or about $10^{-120}$ in reduced Planck units.

\section{Application of Consciousness Model Involving TCP, TRP and $T_{F}$ (Macro) to Explain the Cause for the Expansion of Universe [Having Its Correlation with the Cosmological Constant $(\Lambda)]$}

Einstein gave us his cosmological constant, which he considered a repulsive force equal but opposite to gravity. The majority of scientists today believe this cosmological constant repulsive force does indeed exist between all the stars and galaxies holding them apart.

The cosmological constant is estimated by cosmologists to be on the order of $10^{-29} \mathrm{~g} / \mathrm{cm}^{3}$, or about $10^{-120}$ in reduced Planck units.

Pal et al [3] and Pal [4] expressed that both the TCP and TRP are interchangeable at 'super-symmetry' as they have the same quantized energy and as they behave like biophotons in animals and like simple photons in inanimate objects. These TCP and TRP function like wavicle: wave-particle duality. Pal et al $[1,3]$ have shown that TCP or TRP may be mathematically allotted a mass $\left(\mathrm{m}_{\mathrm{T}}\right)$ equivalent to

$$
4.831 \times 10^{-37} \mathrm{~g} \text { to } 5.5 \times 10^{-37} \mathrm{~g} \text {. }
$$

When $\mathrm{m}_{\mathrm{T}}=$ quantized mass of the TCP $=5.5 \times 10^{-37} \mathrm{~g}$, then

in the case of present universe of $10^{57} \mathrm{~g}$, the calculated number of TCP (in the inherent presence of TRP)

$=10^{57} / 5.5 \times 10^{-37} \cong 1.81818 \times 10^{93} \mathrm{TCP}$ (in the inherent presence of TRP)
It is to be noted that the universe is ultimately made up of $10^{93} \mathrm{TCP}$ (in the inherent presence of TRP), of course, in the presence of Thought force $\left(\mathrm{T}_{\mathrm{F}}\right)$. This thought force $\left(T_{F}\right)$ is an expression of the universal consciousness that, in turn, exists along with the universe. The quantized energy $\left(\varepsilon_{T}\right)$ of the TCP represents universal consciousness.

$$
\mathrm{h} \quad=\text { Reduced Planck constant }=
$$
$h / 2 \pi=6.63 \times 10^{-27}$ erg. sec $/ 2 \times 3.142=1.055 \times 10^{-27}$ erg. sec .

The universe with $10^{93} \mathrm{TCP}$ (in the inherent presence of TRP) gives rise to a value of $\mathrm{h}=1.055 \times 10^{-27} \mathrm{erg}$. sec

$1 \mathrm{TCP}$ (in the inherent presence of TRP) gives rise to a value of $\mathrm{h}=$ $1.055 \times 10^{-27} \mathrm{erg} \cdot \mathrm{sec} / 10^{93} \cong 10^{-120} \mathrm{erg} \cdot \mathrm{sec} \cong 10^{-120} \mathrm{in}$ $\mathrm{h} \cong \Lambda$ (cosmological constant).

Thus $\mathrm{h}$ value of a TCP (in the inherent presence of TRP) in the case of the universe having $1.81818 \times 10^{93} \mathrm{TCP}$ (in the inherent presence of TRP) $\cong 10^{-120} \mathrm{erg}$. sec $\cong 10^{-120}$ in $\mathrm{h} \cong \Lambda$ (cosmological constant).

The functional existence of cosmological constant $(\Lambda)$ signifies the existence of TCP (in the inherent presence of TRP).

Using the upper limit of the cosmological constant, the vacuum energy in a cubic centimeter of free space has been estimated to be $10^{-15}$ Joules as indicated by Carroll [8] $\cong 10^{-16} \mathrm{erg} \cong \mathcal{E}_{T}=$ quantized energy of the TCP. (It is to be noted that $1 e V \cong 1.6021 \times 10^{-12}$ erg $\left.\cong 1.6021 \times 10^{-19} \mathrm{~J}\right)$.

This expression also signifies the existence of TCP (in the inherent presence of TRP) to show the existence and function of cosmological constant $(\Lambda)$.

In 1922, Friedmann of St Petersburg found that Einstein's equations do not permit a static Universe: the universe must be in motion, either expanding or contracting. This contradicted Einstein's belief that the Universe must be eternal in time as well as uniform in space. To remedy this, Einstein introduced the so-called 'cosmological constant' which was physically equivalent to the assumption of a "universal repulsion" between material bodies. In contrast to other physical forces, which always decrease with distance, cosmological repulsion was assumed to be very weak over short distances but important at intergalactic distances. Gamow [9] explained the mathematical expression for this repulsive force acting on a particle of the mass $\mathrm{m}$ as

$$
F=-\left(\frac{1}{3}\right) c^{2} \Lambda m r
$$

where $\quad c=$ velocity of light

$\Lambda=$ Einstein's cosmological constant

$\mathrm{r}=$ distance to the particle.

Dutta [10] indicated that for the Einstein-de Sitter universe the radius $(R)$ of the universe is given as 


$$
R=\frac{2}{(4 \Lambda / 3)^{1 / 2}}
$$

When $R=1.482 \times 10^{29} \mathrm{~cm}$, then it is found that

$\Lambda=$ Einstein's cosmological constant = $3 / R^{2}=1.3659186 \times 10^{-58} \mathrm{~cm}^{-2}$

Again, when $\Lambda=1.3659186 \times 10^{-58} \mathrm{~cm}^{-2}$, $\mathrm{m}=\mathrm{m}_{\mathrm{T}}=$ quantized mass of the TCP $=5.5 \times 10^{-37} \mathrm{~g}$ and

$\mathrm{r}=\mathrm{R}=$ radius of the universe $=1.482 \times 10^{29} \mathrm{~cm}$, then it is found from the Equation (2) that

$$
F=-3.34 \times 10^{-45} \text { dyne }
$$

It is interesting to note that when $\mathcal{E}_{T}=4.95 \times 10^{-16} \mathrm{erg}$ and $D_{i}=R \quad$ (radius of the present universe) $=1.482 \times 10^{29} \mathrm{~cm}$ in the macrocosm, the Equation (1) yields $\mathrm{T}_{\mathrm{F}}($ macro $)=\varepsilon_{T} / D_{i}=3.34 \times 10^{-45}$ dyne.

Thus the value of $\mathrm{F}$ in the Equation (4) is numerically exactly equal to that of $\mathrm{T}_{\mathrm{F}}$ (macro) as shown above.

Further,

$$
\mathrm{T}_{\mathrm{F}}(\text { macro })=\varepsilon_{T} / R=\varepsilon_{T} H_{0} / c=3.34 \times 10^{-45} \text { dyne }
$$

Thus, the universal repulsion $(\mathrm{F})$ is equivalent to $\mathrm{T}_{\mathrm{F}}$ (macro) with a negative sign. If this $\mathrm{T}_{\mathrm{F}}$ (macro) functions as a repulsive force, then it may be found to be responsible for causing the expansion of universe.

As per Gamow [9], the relationship between recession velocity and distance is given by Hubble's law as

[Recession velocity] $=$ constant $\mathrm{x} D$

$$
\text { i.e. } \mathrm{RV}=\mathrm{H}_{\mathrm{o}} \times \mathrm{D}
$$

where $\quad \mathrm{RV}=$ recession velocity

$\mathrm{H}_{\mathrm{o}}=$ Hubble's parameter $=\mathrm{c} / \mathrm{R}$

$\mathrm{D}=$ distance.

Putting $\quad H_{0}=3.34 \times 10^{-45}\left(c / \varepsilon_{T}\right)$ dyne from the Equation (5) in the Equation (6) one can get,

$$
R V=3.34 \times 10^{-45}\left(c / \varepsilon_{T}\right) \text { dynexD }=\left(c / \varepsilon_{T}\right) x T_{F}(\text { macro }) x D
$$

It is thus found that $\mathrm{T}_{\mathrm{F}}$ (macro) is related with the recession velocity $(\mathrm{RV})$. It is further found from the Equation (7) that this $\mathrm{RV}$ is maintained by $\mathrm{T}_{\mathrm{F}}$ (macro) as well as $\mathcal{E}_{T}$, the quantized energy of TCP and c (velocity of light). As c, $\boldsymbol{\varepsilon}_{T}$ and $\mathrm{T}_{\mathrm{F}}$ (macro) exist in this universe through the existence of the quantum mechanical activities of these TCP and TRP, so the universe is liable to expand incessantly through the maintenance of recession velocity in the inherent functional presence of TCP, TRP and $\mathrm{T}_{\mathrm{F}}$ (macro).

\section{Discussion and Conclusion}

\subsection{Discussion}

The cosmological constant is estimated by cosmologists to be on the order of $10^{-29} \mathrm{~g} / \mathrm{cm}^{3}$, or about $10^{-120}$ in reduced Planck units.

It has already been shown that Reduced Planck constant (b) value of a TCP (in the inherent presence of TRP) $\cong 10^{-120} \mathrm{erg}$. sec $\cong 10^{-120}$ in $\mathrm{h} \cong \Lambda$ (cosmological constant).

Using the upper limit of the cosmological constant, the vacuum energy in a cubic centimeter of free space has been estimated to be $10^{-15}$ Joules as indicated by Carroll [8] $\cong 10^{-16} \mathrm{erg} \cong \mathcal{E}_{T}=$ quantized energy of the TCP.

It is the opinion of the majority of scientists today that this cosmological constant repulsive force does indeed exist between all the stars and galaxies holding them apart.

\subsection{Conclusion}

It is shown that the universal repulsion $(\mathrm{F})$ is equivalent to $\mathrm{T}_{\mathrm{F}}$ (macro) with a negative sign. If this $\mathrm{T}_{\mathrm{F}}$ (macro) functions as a repulsive force, then it may be found to be responsible for causing the expansion of universe.

Equation (7) shows that the RV (recession velocity) is related with the $\mathrm{T}_{\mathrm{F}}$ (macro), $\boldsymbol{\varepsilon}_{T}$ (the quantized energy of TCP) and c (velocity of light) and RV is maintained by them. The universe is thus liable to expand incessantly through the maintenance of recession velocity in the inherent functional presence of TCP, TRP and $\mathrm{T}_{\mathrm{F}}$ (macro).

It has already been indicated that the functional existence of cosmological constant $(\Lambda)$ signifies the existence of TCP (in the inherent presence of TRP).

Thus, the consciousness model involving TCP, TRP and $\mathrm{T}_{\mathrm{F}}$ (macro) can provide guidelines to explain the cause for the expansion of universe [having its correlation with the cosmological constant $(\Lambda)]$.

\section{References}

[1] D. Pal and A.U. De, Physics of consciousness and its model may provide guidelines to solve many scientific problems. Neuroquantology 1: 17-28 (2004)

[2] D. Pal, and A.U. De, Consciousness model: Significance of thought-carrying particles and thought-retaining particles in quantum measurement as well as cognitive problem. NeuroQuantology 2: 115-116 (2005)

[3] D. Pal, and A.U. De, The cosmic microwave background radiation temperature signifying the existence of the thought-carrying particle, thought retaining particle and thought force. NeuroQuantology 10: Issue 3; 428-442 (September 2012)

[4] D. Pal, Existence of thought force and its characteristics. Communicated to American Journal of Modern Physics (AJMP) (2013) 
[5] S. Weinberg, Conceptual Foundations of the Unified Theory of Weak and Electromagnetic Interactions. The World of Physics, (Simon and Schuster, 1230 Avenue of Americas, New York 10020) 3: 164 and 165 (1987)

[6] C. Quigg, Elementary Particles and Forces. The World of Physics, (Simon and Schuster, 1230 Avenue of Americas, New York 10020) 2: $884-891$ (1987)

[7] D. H. Perkins, Introduction to High Energy Physics. (Addition-Wesley Publishing Co., Massachusetts 01867, U.S.A) pp. 17 and 364 (1982)
[8] S. Carroll S, C-SPAN broadcast of Cosmology at Yearly Kos Science Panel, Part 1, June 22, (2006)

[9] G. Gamow, The Great Expansion. The World of Physics. (Simon and Schuster, 1230 Avenue of Americas, New York 10020) 3: 261-264 (1987)

[10] S. K. Dutta, The Noninfinite, Nonzero Quantized Energy Limits and Their Physical Significance. Physics Essays. 8, number 4, 469 (1995) 